the peculiar position in which he stands." Clinical scientists could well use this maxim as their guide in constructing the best clinical trial for the condition they wish to study.

${ }^{1}$ Jones, F E, Biomedicine, 1978, 28, 55.

2 Huguley, C M, et al, Cancer, 1975, 36, 1227

${ }^{3}$ Rimm, A A, and Bortin, M, Biomedicine, 1978, 28, 60.

+ Dudley, H, British Medical fournal, 1978, 1, 1344.

Gehan, E A, Biomedicine, 1978, 28, 13.

\section{Drug administration and food}

Most drugs are given by mouth-a route that is convenient and usually safe for the patient and makes no demands on medical time. Nevertheless, the amount of the drug that reaches the systemic circulation unchanged after oral intake is variable. In contrast with the certainty after an intravenous injection, this variable fraction is referred to as absolute bioavailability and depends on many factors. ${ }^{1}$

The formulation of the drug determines the rates of both disintegration and dissolution. Physicochemical characteristics are also important and include the crystalline structure of the preparation and the extent of ionisation of the drug molecule at the $\mathrm{pH}$ of the intestine. Highly ionised drugs such as the quaternary ammonium compounds are poorly and unreliably absorbed because they cannot cross cell membranes by passive diffusion. In contrast, non-ionised drugs which readily dissolve in lipid-propranolol, for example-may be almost completely absorbed from the gut. ${ }^{2}$ Gastric motility determines the rate of delivery of the drug to the small intestine. If the medicine is in solution by the time it reaches the small bowel absorption will be rapid and may be complete. In contrast, if gastric emptying is rapid and the drug dissolves comparatively slowly, rapid gastrointestinal transit may reduce bioavailability. Finally, bioavailability may be lowered by acid hydrolysis of the drug (for example, benzyl penicillin) or by its degradation by intestinal or hepatic enzymes. Biotransformation by such enzymes during absorption is often referred to as "metabolism at the first-pass."

Despite recent improvements in the information provided about drugs, comparatively few data sheets say whether the product should be taken with food or on an empty stomachthough food may have any one of several effects on the processes which determine bioavailability. Most studies have been based on single doses of drugs, and we need more work on patients taking regular medication. Nevertheless, the range of drugs whose bioavailability is improved by concurrent administration with food has been reviewed recently by Melander ${ }^{3}$; it includes the hypotensives hydrallazine, hydrochlorothiazide, and canrenone (the major metabolite of spironolactone) and possibly also the $\beta$-adrenoceptor antagonists propranolol and metoprolol. In contrast, quaternary ammonium compounds are less reliably absorbed in the presence of food, and this applies also to many antibiotics, particularly tetracyclines, ampicillin (but not amoxycillin), and the antituberculous drugs isoniazid and rifampicin. On the other hand, the absorption of nitrofurantoin is improved by concurrent administration with food.

How is absorption of drugs improved when they are given with meals? Among the possible explanations are alterations in tablet disintegration and drug dissolution and the variable effects of different types of meals on transit times through the gastrointestinal tract. Possibly, too, substances present in the food may affect the activity of enzymes located in the gut mucosa and liver. For inhibitors such actions would be immediate, but for other substances which induce microsomal enzyme oxidation the effect is likely to be seen only during regular exposure. One ingenious explanation recently proposed for the reported improved bioavailability of propranolol and metoprolol when given with food ${ }^{4}$ is that the stimulus of a meal might increase splanchnic blood flow and so improve the bioavailability of drugs (like propranolol) which undergo extensive metabolism at the first-pass through the liver. ${ }^{5}$ Using a computer model McLean et al $l^{5}$ produced findings consistent with this hypothesis-but all computer simulations are as good only as the programme used and the data supplied. Certainly the extraction of propranolol across the liver is dependent on blood flow, ${ }^{6}$ and hepatic blood flow is a major determinant of the half life of the drug when given intravenously. Nevertheless, the changes in the extraction ratio are relatively small with quite large variations in the hepatic blood flow. Furthermore, Wilkinson and Shand ${ }^{7}$ have shown that the bioavailability of propranolol given by mouth is chiefly related to the activity of microsomal drug-oxidising enzymes in the liver. While the bioavailability of drugs might be affected by changes in splanchnic blood flow after food, their proposal must await confirmation by careful studies.

Clearly we need to know more about drug administration and food, particularly during regular dosing. Food, its components and contaminants, probably has both short-term and long-term effects on absorption and biotransformation. The net effect on drug bioavailability cannot be predicted but must be measured in direct clinical studies of the drugs and foods in question. Furthermore, we may need to look closer at our current practice of studying drug bioavailability in patients and normal volunteers when they are fasting, for these may not represent the way in which the drug will be used in clinical practice. Perhaps, therefore, we should do our pharmacokinetic studies on new drugs under both fasting and nonfasting conditions using standardised test meals.

${ }^{1}$ George, C F, British Medical fournal, 1976, 2, 742.

2 Paterson, J W, European fournal of Clinical Pharmacology, 1970, 2, 127.

${ }^{3}$ Melander, A, Clinical Pharmacokinetics, 1978, 3, 337.

${ }^{4}$ Melander, A, Clinical Pharmacology and Therapeutics, 1977, 22, 108.

${ }^{5}$ McLean, A J, et al, Clinical Pharmacology and Therapeutics, 1978, 24, 5.

6 Weiss, Y A, et al, British fournal of Clinical Pharmacology, 1978, 5, 457.

7 Wilkinson, G R, and Shand, G D, Clinical Pharmacology and Therapeutics, $1975,18,377$.

\section{Fifty years of gold in rheumatoid arthritis}

Fifty years of treatment with gold in rheumatoid arthritis have elapsed-an anniversary that has been almost unnoticed. Jacques Forestier first used gold salts in rheumatoid arthritis in 1928 on an empirical basis, as they had appeared to have some effect on tuberculosis. ${ }^{1}$ Since then the popularity of gold has waned and waxed several times, but the amount used in Britain has increased steadily ever since the Empire Rheumatism Council report in 1961 finally established the clinical benefit of gold in a long-term controlled trial. ${ }^{2}$ It remains the last of the heavy metals in common clinical use, though we know little more about the reason for its effectiveness now than 50 years ago.

Nowadays rheumatoid arthritis is carefully defined, and 
many diseases once thought to be rheumatoid are recognised as separate entities. Among these are systemic lupus erythematosus and the seronegative spondarthroses (in particular, ankylosing spondylitis)-entities in which treatment with gold is at worst potentially harmful and at best usually unhelpful. The side effects ascribed to gold have also changed: jaundice has virtually disappeared since the introduction of disposable needles and syringes; proteinuria and the nephrotic syndrome are usually recognised to be due to secondary amyloidosis. Cutaneous manifestations remain a problem, but are now seen as a reason for temporary discontinuation rather than as an absolute contraindication to treatment. Bone marrow depression, occasionally fatal, still occurs; but clinicians are now more aware of the problem, and with careful monitoring ${ }^{3}$ the number of deaths reported annually to the Committee on Safety of Medicines has not paralleled the increased use of gold. ${ }^{4}$

Methods of prescribing gold are still changing. Once it was given as four injections each of $250 \mathrm{mg}$. In the Empire Rheumatism Council controlled trial the course was of 20 injections of $50 \mathrm{mg}$. In the period of surveillance after that trial many patients relapsed, and monthly maintenance injections were introduced. A fixed-dosage regimen of gold on this pattern was compared with flexible regimens of cytotoxic drugs and with penicillamine..$^{5}$ In the last few years ideas have changed again, and flexible regimens have been tailored to the patients' needs ${ }^{7}$ with improved results and less toxicity. When gold is used earlier in the course of the disease $^{8}$ remissions will occur sooner; and in these circumstances the frequency of injections should be reduced ${ }^{7}$ at the time of clinical remission to lessen the risk of toxicity.

At the end of 50 years the consensus is that gold is still useful in rheumatoid arthritis. The recent introduction of a new effective oral gold preparation may change our ideas again. ${ }^{9}$ And it is always possible that 1979 may see the discovery of a real cure for rheumatoid arthritis-or of its cause.

${ }^{1}$ Forestier, J M, Bulletin et Mémoires de la Société Médicale des Hôpitaux de Paris, 1929, 53, 323.

2 Research Subcommittee of the Empire Rheumatism Council, Annals of the Rheumatic Diseases, 1961, 20, 315.

${ }^{3}$ Kay, A G L, British Medical fournal, 1976, 1, 1266.

4 Gumpel, J M, British Medical fournal, 1978, 1, 215.

5 Woodland, J, et al, Annals of the Rheumatic Diseases, 1974, 33, 399.

${ }^{6}$ Huskisson, E C, et al, Annals of the Rheumatic Diseases, 1974, 33, 532.

7 Gumpel, J M, Rheumatology and Rehabilitation, 1976, 15, 217.

${ }^{8}$ Luukkainen, R, Kajander, A, and Isomäki, H, Scandinavian fournal of Rheumatology, 1977, 6, 189.

${ }^{9}$ Finkelstein, A E, et al, Annals of the Rheumatic Diseases, 1976, 35, 251.

\section{Preventing endocarditis}

Last year the American Heart Association made revised recommendations for antibiotic cover of procedures known to cause bacteraemia. ${ }^{1}$ These include not only dental extraction and other surgery in the mouth and throat but various operations on the urinary and lower alimentary tracts. Another procedure-namely, transrectal biopsy of the prostate-has recently joined the list. ${ }^{2}$ The new recommendations evidently owed much to the experimental studies of D T Durack, a member of the committee formulating them, who argued from his results that only a large and preferably parenteral dose of a bactericidal antibiotic or combination of antibiotics is adequate. The regimen proposed for dental operations is a large parenteral dose of penicillin followed by eight doses of $0.5 \mathrm{~g}$ penicillin $\mathrm{V}$ at 6-hour intervals or, in patients allergic to penicillin, intravenous vancomycin followed by a similar course of erythromycin. To guard against infection by the more resistant enterococcus in abdominal operations, the proposed cover is even more formidable-penicillin or ampicillin plus gentamicin or streptomycin given parenterally in three doses.

We commented that these proposals placed a formidable responsibility on those obeying them, and suggested that Durack's experimental model was too severe a test for his conclusions to apply literally to transient bacteraemia in man. ${ }^{3}$ It consisted of producing vegetations on the aortic valves of rabbits by inserting catheters into the cardiac chambers and inducing bacteraemia with a massive intravenous injection of streptococci. The criterion for adequate treatment was sterilising the blood in 24 hours. We questioned this model on the ground that, as bacteria on these vegetations may be less accessible than those on the intact endothelial surface of even a valve deformed by disease, this type of experiment is to some extent an exercise in treating rather than preventing endocarditis; and 24 hours may be too short a time in which to expect sterilisation.

This criticism of the method has now been echoed by Petersdorf ${ }^{4}$-even though he was co-author with Durack of the two principal accounts of these experiments. ${ }^{5} \mathrm{He}$ calls his paper "Antimicrobial prophylaxis of bacterial endocarditis: prudent caution or bacterial overkill ?" apparently having reacted against the "hyperaggressive recommendations" and particularly those of Kaye, ${ }^{7}$ who proposes even larger doses of the same parenteral antibiotics continued for three days. Petersdorf points out not only that patients dislike injections and that "intravenous therapy with a drug like vancomycin in the dentist's office seems totally impractical," but that Kaye's proposals call for three days in hospital and could cost as much as $\$ 1000$. His own proposals are much simpler. Parenteral prophylaxis against Streptococcus viridans endocarditis, with a single dose of procaine penicillin and streptomycin, "should be reserved for high-risk patients such as those with prosthetic heart valves, and should not be used for patients without such intracardiac devices." This alone among his suggestions is disputable: is not an existing rheumatic valvular defect or even a clear history of rheumatic fever another indication-not to mention a previous attack of bacterial endocarditis? For "most other patients" he suggests a $2 \mathrm{~g}$ oral dose of penicillin $\mathrm{V}$ followed by three doses of $0.5 \mathrm{~g}$ or similar treatment with erythromycin if the patient is allergic to penicillin-emphasising the need for only three rather than eight further doses. Parenteral treatment is needed, however-as he acknowledges - to prevent enterococcal infection, and he recommends penicillin or ampicillin plus gentamicin. The treatment could be completed in 12 to 24 hours.

The observations of Shanson and his colleagues, who made blood cultures from treated and control patients at the London Hospital two minutes after dental extraction, should encourage us to rely on a single large oral prophylactic dose of antibiotic. ${ }^{8}$ In the past such cultures have often proved little, because antibiotic in the culture could have prevented the growth of surviving bacteria. But Shanson added penicillinase to one of two bottles after four hours' incubation and to the other after 24 hours, so that any surviving bacteria could multiply. There were 40 patients in each of three groups. Aerobic streptococci grew from the blood of 14 controls and from five given penicillin $\mathrm{V}$ and two given amoxycillin (each in a dose of $2 \mathrm{~g}$ one hour before extraction). These growths were in bottles to which penicillinase had been added after four hours; when it 